\title{
Role of cyclin polymorphisms in predicting outcome of 5-fluorouracil-based chemotherapy in colorectal cancer: one piece in a complex puzzle
}

\begin{abstract}
"While the insight provided from studying the cell cycle and its associated proteins has advanced our understanding of the complex network and crossover between signaling pathways in both normal and cancer cells and has led to new drug targets and novel combination chemotherapies, the true importance of these molecules in mediating drug response still remains to be fully established."
\end{abstract}

\section{KEYWORDS: 5-FU $\approx$ apoptosis $\approx$ CDK inhibitors $\approx$ cell cycle = colorectal cancer $\approx$ cyclin D " DNA damage " polymorphism $\approx$ thymidylate synthase}

The last 20 years has witnessed a significant increase in our knowledge and understanding of the complex pathways and molecular signals that are deregulated in colorectal cancer (CRC). This remarkable progress has been made possible through numerous technological advances and extensive collaborative efforts within the pharmaceutical, clinical and research communities and has led to the identification of promising new drug targets and the development of new drugs. However, despite this progress, CRC has been plagued with a high rate of drug development failure and progress remains largely incremental. Surprisingly, for the last 50 years, the antimetabolite 5-fluorouracil (5-FU) remains the most effective and widely used chemotherapeutic agent for the treatment for CRC.

5-FU functions primarily through the inhibition of the DNA biosynthetic enzyme TS. The TS enzyme catalyzes the reductive methylation of deoxyuridine monophosphate to thymidine monophosphate, a critical step in generating thymine, one of the four nucleoside triphosphates essential for the in vivo synthesis of DNA. As TS represents the sole intracellular source of de novo thymidine monophosphate, the inhibition of this enzyme exploits one of the few bottlenecks in the DNA synthesis pathway and serves as a critical target in cancer chemotherapy. As the primary mechanism of 5-FU is disruption of DNA synthesis, it is highly active against replicating cells where the rapid depletion of deoxythymidine triphosphate pools and subsequent perturbations in the levels of the other deoxynucleotides severely disrupts DNA synthesis and repair, resulting in cell cycle arrest, DNA damage and apoptosis [1]. However, DNA damage occurs in all phases of the cell cycle and in normal cells is detected by various checkpoints at $G_{1}-S, S$ or $G_{2}-M$, resulting in inhibition of cell cycle progression to allow for repair of damage and maintenance of genomic stability [2]. Significant research has focused on determining the optimum 5-FU dose and administration schedule to maximize efficacy and manage toxicity. Research reports have demonstrated that 5-FU has different antitumor effects based on the dose administered: either a $G_{1}-S$ cell cycle arrest or a $G_{2}-M$ cell cycle arrest coupled with mitotic catastrophe [3]. In the clinic, improvements to 5-FU efficacy have been achieved through enhancing TS inhibition by incorporating the reduced folate leucovorin into 5-FU regimens. In addition, continuous infusion (vs bolus injection) has demonstrated improved clinical outcome in CRC attributed to a more consistent inhibition of TS. These key changes in 5-FU regimens have demonstrated that tangible improvements to clinical efficacy can be achieved through an increased understanding of the function of 5-FU at the molecular level. However, a large percentage of patients treated with 5-FU-based therapy do not respond and understanding the molecular factors determining sensitivity or resistance to 5-FU at the cell cycle level may represent a critical step in continuing to improve the clinical impact of this pivotal therapy.

Genomic integrity and DNA damage are inextricably linked to cell cycle control. Therefore, one of the mechanisms of tumor resistance to 5-FU has been attributed to abnormalities and a breakdown within cell cycle control. In normal cells, the detection of DNA damage as a result of 5-FU treatment results in the induction of the DNA damage response, rapid cell cycle arrest and in some instances apoptosis. Aberrant

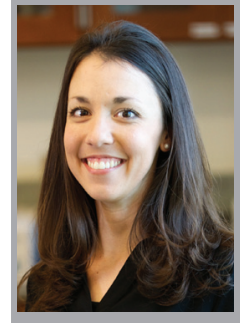

Melissa J LaBonte Department of Biology \& Chemistry, Azusa Pacific University, 901 East Alosta Avenue, Azusa, CA, USA

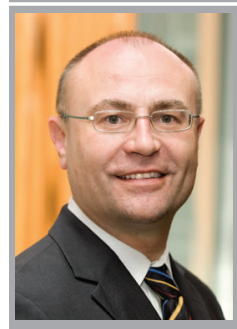

Heinz-Josef Lenz Author for correspondence: Division of Medical Oncology, Norris Comprehensive Cancer Center, Keck School of Medicine, University of Southern California, Los Angeles, CA, USA lenz@usc.edu 
functioning of cell cycle checkpoints in some tumors has the potential to interfere with and greatly diminish 5-FU cytotoxicity. Consistent with this, previous studies have identified key roles for cell cycle progression/regulatory proteins in determining tumor sensitivity to 5-FU [4]. Numerous studies have been conducted to analyze the expression of genes, genetic variation (i.e., copy number and SNPs) and the epigenetic modification (i.e., methylation and acetylation) of key proteins that contribute to cell cycle control, including cyclins, cyclin dependent kinases (CDKs) and checkpoint kinases. The goal of these studies is to identify potential new targets for therapeutic intervention and to implement patient stratification according to molecular profiles to enhance the activity of 5-FU in CRC patients [2,5-8].

\section{"With regards to understanding and predicting individual patient response to 5-fluorouracil-based therapy, the cell cycle proteins including cyclins such as cyclin D1 are only one piece of what appears to be a complex puzzle."}

The protein complexes that are responsible for regulating cell cycle progression are composed of CDKs (catalytic serine/threonine kinase subunits) and cyclin (activating subunits) that exert their regulatory role at specific phases of the cell cycle $\left(G_{1}\right.$ : cyclin D/CDK4/6; $G_{1} / S$ : cyclin E/CDK2; S: cyclin A/CDK1/2; G $/ \mathrm{M}$ : cyclin B/CDK1) [9]. These complexes are regulated by mitotic inhibitors that inhibit the $\mathrm{G}_{1}$ checkpoint: p15, p16 ${ }^{\mathrm{INK} 4}, \mathrm{p} 18$ and p19; and the $G_{1} / S$ and $S$ checkpoints: p21 and p27 ${ }^{\mathrm{KIP} 1}$, p2 $7^{\text {WAF1/CIP1 }}$ and p57 $7^{\mathrm{KIP} 2}[10]$. Cyclin levels oscillate in a regulated fashion during the cell cycle through fluctuations in gene expression and degradation via the ubiquitin-mediated proteasome pathway. When cyclins are present, they complex with their specific CDKs that are subsequently phosphorylated, thus allowing the cell to enter the next phase of the cell cycle.

In normal cells, the checkpoints and cyclin/CDK complexes facilitate the recognition of abnormal cellular conditions and potential risks to DNA integrity, triggering cell cycle arrest in conditions such as DNA damage, organelle stress (such as endoplasmic reticulum stress) or mitotic complications. However, cancer cells develop abnormalities and mutations in both the DNA damage/repair and cell cycle checkpoint pathways that lead to an increased likelihood of survival and continued proliferation in the presence of DNA damage and cellular stress. The resulting DNA damage serves to further increase genetic abnormalities, fuel malignant progression and decreases the cancer cell's ability to exit the cell cycle to a dormant state in response to growth inhibitory signals. The status of both the DNA damage repair and cell cycle checkpoint pathways can have profound effects on how a cancer cell responds to chemotherapies that induce DNA damage and inhibit DNA replication.

Several cell cycle checkpoint proteins that are most frequently altered in CRC are involved in controlling the $G_{1} / S$ transition and include the $\mathrm{p} 16^{\mathrm{INK} 4 \mathrm{~A}} / \mathrm{cyclin} \mathrm{D} 1 /$ retinoblastoma/E2F pathway $[11,12]$. Owing to the critical function of these pathways in driving tumor growth and survival, significant research has been directed toward cataloging mutations, measuring expression and identifying the presence of genetic polymorphisms in the genes within these pathways in an effort to identify the underlying cause of the significant variability to chemotherapy in numerous cancer types including CRC. Results from some of these studies have identified potential new therapeutic targets within the cell cycle machinery, mainly CDKs that have since led to the development of novel chemotherapeutics.

Several retrospective studies in CRC have analyzed an important SNP in cyclin D1 (CCND1, rs9344 $\mathrm{G}>\mathrm{A}$ ) and have demonstrated associations with clinical outcome. Cyclin D1, a member of the D-type cyclin family and an essential regulator of cell cycle progression of the $G_{1} / S$ phase, is also a well-documented oncogene whose increased expression has been firmly linked to the etiology of numerous solid tumors. Two mRNA transcripts have been identified for cyclin D1, transcript-a and transcript-b with the latter being associated with the variant $\mathrm{A}$ allele. This transcript-b lacks aproline-glutamic acid-serine-theonine region critical for destabilizing CCND1 and it's loss leads to a significant increase in the variant protein half-life. The rs 9344 SNP at position 870 occurs directly at the donor splice site between exon 4 and 5 and patients homozygous for the $\mathrm{G}$ allele, demonstrate predominant tumoral expression of transcript-a and the full-length wild-type cyclin D1 protein with normal activity. Patients homozygous for the A allele demonstrate elevated tumoral expression of the alternatively spliced mRNA transcript-b with increased cyclin D1 activity and associated oncogenic functions. Heterozygous patients express both transcripts with intermediate cyclin D1 activity [13,14]. Importantly, transcript-a is associated with an 
enhanced DNA damage response and increased apoptosis in response to DNA damage [15]. Consistent with this, a positive association between the AA genotype and a decreased time-to-tumor recurrence in patients treated with 5-FU-based chemotherapy was recently reported in stage 2 and 3 CRC in two independent cohorts comprising of over 400 patients treated with 5-FU-based therapy. This difference in time to tumor recurrence with A870G was observed only in patients treated with 5-FU-based therapy and not those treated with surgery alone, suggesting a predictive role over any prognostic effect in these patients. These results suggest that the normal functions of cyclin D1 that are compromised in transcript-b encoded by the A allele such as activation of the DNA damage response are important mediators of 5-FU cytotoxicity [16]. This study is in agreement with another recent study that identified the same detrimental effect of the A allele in breast cancer patients treated with the 5-FU prodrug capecitabine. These studies provide insight into the critical pathways responsible for individual drug action and combination chemotherapeutic regimens that are administered to CRC patients. Interestingly, the observation that SNPs in cyclin D1 have been associated with predicting tumor responses to both 5-FU-based and cetuximab-containing combinations suggests that inhibition of these pathways may converge on a common cell cycle pathway, helping explain their synergistic interaction.

In addition to analysis of cyclin D, CDK4 and 6 have been developed as drug targets owing to their interaction with cyclin $\mathrm{D}$ to inactivate $\mathrm{Rb}$ and enable cell cycle progression from $\mathrm{G}_{1}$ to $S$ phase. Several small molecule inhibitors have been developed against CDK4 and 6 and when tested in clinical trials demonstrated little activity as single agents in solid tumors, including CRC, but show promise when combined with cytotoxic chemotherapies $[17,18]$.

While the insight provided from studying the cell cycle and its associated proteins has advanced our understanding of the complex network and crossover between signaling pathways in both normal and cancer cells and has led to new drug targets and novel combination chemotherapies, the true importance of these molecules in mediating drug response still remains to be fully established. With regards to understanding and predicting individual patient response to 5-FU-based therapy, the cell cycle proteins including cyclins such as cyclin D1 are only one piece of what appears to be a complex puzzle. It is likely that the key to predicting response to 5-FU-based therapy will require rigorous analysis of pathways involving drug metabolism, DNA damage and repair as well as cell cycle and apoptotic pathways. The role of the cyclin D1 rs9344 polymorphism needs to be validated in additional cohorts and further analyzed in a prospective randomized trial to provide evidence for its predictive role [5].

\section{Financial \& competing interests disclosure}

The authors have no relevant affliations or financial involvement with any organization or entity with a financial interest in or financial conflict with the subject matter or materials discussed in the manuscript. This includes employment, consultancies, honoraria, stock ownership or options, expert testimony, grants or patents received or pending, or royalties.

No writing assistance was utilized in the production of this manuscript.

\section{References}

1 Longley DB, Harkin DP, Johnston PG. 5-fluorouracil: mechanisms of action and clinical strategies. Nat. Rev. Cancer 3(5), 330-338 (2003).

2 Zhou BB, Bartek J. Targeting the checkpoint kinases: chemosensitization versus chemoprotection. Nat. Rev. Cancer 4(3), 216-225 (2004).

3 Yoshikawa R, Kusunoki M, Yanagi $\mathrm{H}$ et al. Dual antitumor effects of 5-fluorouracil on the cell cycle in colorectal carcinoma cells: a novel target mechanism concept for pharmacokinetic modulating chemotherapy. Cancer Res. 61(3), 1029-1037 (2001).

4 Guo X, Goessl E, Jin G et al. Cell cycle perturbation and acquired 5-fluorouracil chemoresistance. Anticancer Res. 28(1A), 9-14 (2008).
5 Labonte MJ, Wilson PM, Yang D et al. The Cyclin D1 (CCND1) A870G polymorphism predicts clinical outcome to lapatinib and capecitabine in HER2-positive metastatic breast cancer. Ann. Oncol. 23(6), 1455-1464 (2012).

6 Macaluso M, Montanari M, Cinti C, Giordano A. Modulation of cell cycle components by epigenetic and genetic events. Semin. Oncol. 32(5), 452-457 (2005).

7 Myklebust MP, Li Z, Tran TH et al. Expression of cyclin D1a and D1b as predictive factors for treatment response in colorectal cancer. Br. J. Cancer 107(10), 1684-1691 (2012).

8 Chung H, Chaudhry J, Lopez CG, Carethers JM. Cyclin E and histone $\mathrm{H} 3$ levels are regulated by 5 -fluorouracil in a DNA mismatch repair-dependent manner. Cancer Biol. Ther. 10(11), 1147-1156 (2010).
9 Kastan MB, Bartek J. Cell-cycle checkpoints and cancer. Nature 432(7015), 316-323 (2004).

10 Rahman MM, Kipreos ET. The specific roles of mitotic cyclins revealed. Cell Cycle 9(1), 22-23 (2010).

11 Veganzones-de-Castro S, Rafael-Fernandez $\mathrm{S}$, Vidaurreta-Lazaro $\mathrm{M}$ et al. p16 gene methylation in colorectal cancer patients with long-term follow-up. Rev. Esp. Enferm. Dig. 104(3), 111-117 (2012).

12 Ogino S, Nosho K, Irahara N et al. A cohort study of cyclin D1 expression and prognosis in 602 colon cancer cases. Clin. Cancer Res. 15(13), 4431-4438 (2009).

13 Knudsen KE. The cyclin D1b splice variant: an old oncogene learns new tricks. Cell Div. 1, 15 (2006).

14 Solomon DA, Wang Y, Fox SR et al. Cyclin D1 splice variants. Differential effects on 
localization, RB phosphorylation, and cellular transformation. J. Biol. Chem. 278(32), 30339-30347 (2003).

15 Li Z, Jiao X, Wang C et al. Alternative cyclin D1 splice forms differentially regulate the DNA damage response. Cancer Res. 70(21), 8802-8811 (2010).

16 Absenger G, Benhaim L, Szkandera J et al. The cyclin D1 (CCND1) rs9344 G>A polymorphism predicts clinical outcome in colon cancer patients treated with adjuvant 5-FU-based chemotherapy.

Pharmacogenomics J. doi:10.1038/tpj.2013.15 (2013) (Epub ahead of print).

17 Flaherty KT, Lorusso PM, Demichele A et al. Phase I, dose-escalation trial of the oral cyclin-dependent kinase $4 / 6$ inhibitor PD
0332991, administered using a 21-day schedule in patients with advanced cancer. Clin. Cancer Res. 18(2), 568-576 (2012).

18 Aklilu M, Kindler HL, Donehower RC, Mani $S$, Vokes EE. Phase II study of flavopiridol in patients with advanced colorectal cancer. Ann. Oncol. 14(8), 1270-1273 (2003). 